\title{
A study of compressibility and compactibility of directly compressible tableting materials containing tramadol hydrochloride
}

\author{
JITKA MUŽÍKOVÁ* \\ ALENA KUBÍČKOVÁ \\ Department of Pharmaceutical \\ Technology \\ Charles University in Prague \\ Faculty of Pharmacy \\ in Hradec Králové \\ Czech Republic
}

Accepted January 11, 2016

Published online April 26, 2016

\begin{abstract}
The paper evaluates and compares the compressibility and compactibility of directly compressible tableting materials for the preparation of hydrophilic gel matrix tablets containing tramadol hydrochloride and the coprocessed dry binders Prosolv ${ }^{\circledR}$ SMCC 90 and Disintequik ${ }^{\mathrm{TM}}$ MCC 25. The selected types of hypromellose are Methocel ${ }^{\mathrm{TM}}$ Premium K4M and Methocel ${ }^{\mathrm{TM}}$ Premium K100M in 30 and $50 \%$ concentrations, the lubricant being magnesium stearate in a $1 \%$ concentration. Compressibility is evaluated by means of the energy profile of compression process and compactibility by the tensile strength of tablets.

The values of total energy of compression and plasticity were higher in the tableting materials containing Prosolv ${ }^{\circledR}$ SMCC 90 than in those containing Disintequik ${ }^{\mathrm{TM}}$ MCC 25. Tramadol slightly decreased the values of total energy of compression and plasticity. Tableting materials containing Prosolv ${ }^{\circledR}$ SMCC 90 yielded stronger tablets. Tramadol decreased the strength of tablets from both coprocessed dry binders.
\end{abstract}

Keywords: tramadol hydrochloride, hydrophilic matrix tablets, coprocessed dry binder, hypromellose, energy profile of compression, tensile strength of tablets

Dosage forms with prolonged release of the active ingredient represent a contemporary trend, prolonged-release tablets being an important form. One of its technological types are matrix tablets, in which the active ingredient including the retarding component is distributed throughout the whole matrix of the tablet. According to the type of the retarding component, matrix tablets are classified as polymeric insoluble, hydrophilic gel, lipophilic, or mixed (1). The simplest method of producing matrix tablets is direct compression. Direct compression requires excipients called dry binders, which fulfil the function of filler and binder at the same time (2). Several dry binders are sometimes present, or the dry binder and another excipient are combined to form one substance by the so-called coprocessing and give rise to coprocessed dry binders. These substances are aimed at increasing the

\footnotetext{
*Correspondence; e-mail: muzikova@faf.cuni.cz
} 
positive properties of individual ingredients and suppressing the negative ones. They may also be of multifunctional character (3). Dry binders include also substances considered in this study, i.e., Prosolv ${ }^{\circledR}$ SMCC 90 and Disintequik ${ }^{\text {TM }}$ MCC 25. Prosolv ${ }^{\circledR}$ SMCC is silicified microcrystalline cellulose (SMCC) containing $98 \%$ of microcrystalline cellulose (MCC) and $2 \%$ of colloidal silicon dioxide in a patented homogeneous mixture. It shows some advantages, such as good flow properties, a short period of disintegration, and high mechanical strength of tablets resulting from the intersurface interaction of silicon dioxide and MCC $(4$, 5). In coprocessing, colloidal silicon dioxide is uniformly dispersed over microcrystalline cellulose and forms a rough surface of SMCC in comparison with MCC, the surface of which is much smoother and smaller (6). Prosolv ${ }^{\circledR}$ SMCC 90 contains particles of an average size of $110 \mu \mathrm{m}$, which ensures good flowability and also suitability for direct compression (7). Disintequik $^{\mathrm{TM}}$ MCC 25 is a relatively new coprocessed dry binder containing $75 \%$ of $\alpha$-lactose monohydrate and $25 \%$ of microcrystalline cellulose (8). This combination of substances is employed because of their synergic effect, which consists of greater strength and rapid disintegration of tablets. The lactose present is a fragile material, in which fragmentation takes place, thus providing areas for the development of new bonds. MCC is characterized by plastic deformation and enables rapid disintegration of tablets (9). Disintequik ${ }^{\mathrm{TM}}$ MCC 25 is characterized by better flow properties, which support mass uniformity of tablets and better compressibility than in the physical mixture of lactose and microcrystalline cellulose (8). Hydrophilic gel matrix tablets also contain an auxiliary gel-forming substance, which decelerates the release of the active ingredient. This excipient is very often hypromellose, which comes in several viscosity degrees (10). Examples are the products of Methocel $^{\mathrm{TM}}$ Premium K4M and K100M, which were employed in this study.

The aim of this paper was to study compressibility and compactibility of directly compressible tableting materials intended for preparation of hydrophilic gel matrix tablets with tramadol hydrochloride. Tableting materials tested included two types of coprocessed dry binders and two viscosity types of hypromellose in concentrations of 30 and 50 $\%$. Compressibility was evaluated by means of the energy profile of the compression process and compactibility by the tensile strength of tablets.

\section{EXPERIMENTAL}

\section{Materials}

Silicified microcrystalline cellulose Prosolv ${ }^{\circledR}$ SMCC 90 was supplied by JRS Pharma, GmbH+Co.kG (Germany), Disintequik ${ }^{\mathrm{TM}}$ MCC 25 by Kerry (USA), hypromellose (Metho$\mathrm{cel}^{\mathrm{TM}} \mathrm{K} 4 \mathrm{M}$ Premium CR, Methocel ${ }^{\mathrm{TM}} \mathrm{K} 100 \mathrm{M}$ Premium CR) by the firm Colorcon GmbH (Germany). Magnesium stearate was obtained from Acros Organics (USA). Tramadol hydrochloride was from Sigma-Aldrich (Czech Republic).

\section{Preparation of tableting materials}

Altogether sixteen tableting materials of the following compositions were prepared and tested (Table I).

Tableting materials were prepared using a mixing cube KB $15 S$ (Erweka GmbH, Germany). The mixing rate was $17 \mathrm{rot} / \mathrm{min}$. The appropriate type of Methocel $^{\mathrm{TM}}$ with Prosolv ${ }^{\circledR}$ 
Table I. Composition of tableting materials (\%)

\begin{tabular}{|c|c|c|c|c|c|c|}
\hline $\begin{array}{l}\text { Tableting } \\
\text { material }\end{array}$ & P 90 & D 25 & Tramadol & M4M & M100M & Mgst \\
\hline 1 & 49 & & 20 & 30 & & 1 \\
\hline 2 & 29 & & 20 & 50 & & 1 \\
\hline 3 & 49 & & 20 & & 30 & 1 \\
\hline 4 & 29 & & 20 & & 50 & 1 \\
\hline 5 & 69 & & & 30 & & 1 \\
\hline 6 & 49 & & & 50 & & 1 \\
\hline 7 & 69 & & & & 30 & 1 \\
\hline 8 & 49 & & & & 50 & 1 \\
\hline 9 & & 49 & 20 & 30 & & 1 \\
\hline 10 & & 29 & 20 & 50 & & 1 \\
\hline 11 & & 49 & 20 & & 30 & 1 \\
\hline 12 & & 29 & 20 & & 50 & 1 \\
\hline 13 & & 69 & & 30 & & 1 \\
\hline 14 & & 49 & & 50 & & 1 \\
\hline 15 & & 69 & & & 30 & 1 \\
\hline 16 & & 49 & & & 50 & 1 \\
\hline
\end{tabular}

P 90 - Prosolv SMCC 90, D 25 - Disintequik MCC 25, M4M - Methocel K4M Premium CR, M100M-Methocel K100M Premium CR, Mgst - magnesium stearate

SMCC 90 or Disintequik ${ }^{\mathrm{TM}}$ MCC 25 was first mixed for a period of 5 minutes. Tramadol was then added for another 5 minutes, and finally magnesium stearate was added for a mixing period of 2.5 minutes. Mixtures without the active ingredient were prepared in the same manner without the mixing step with tramadol, their mass being $20 \mathrm{~g}$. The mass of tableting materials with tramadol was $30 \mathrm{~g}$.

\section{Preparation of tablets and energy evaluation of compression process}

Tablets were compressed using a material testing equipment T1-FRO 50 TH.A1K Zwick/Roell (Zwick GmbH \& Co, Germany) by means of a special die with a lower and an upper punch. The rate of compaction was $40 \mathrm{~mm} \mathrm{~min}^{-1}$, pre-load was $2 \mathrm{~N}$, and the rate of preload was $2 \mathrm{~mm} \mathrm{~s}^{-1}$. The tablets were of cylindrical shape without facets, with a $13 \mathrm{~mm}$ diameter, $m=0.5000 \pm 0.0010 \mathrm{~g}$. The employed compression force was $5 \mathrm{kN}$. At this compression force, 10 tablets were compressed. The computer program testXpert V 9.01 (Zwick $\mathrm{GmbH \& Co}$ ) simultaneously recorded the energy process of compression by means of the „force-displacement" record and numerically evaluated the energy balance of compression, i.e., the energy of precompression $E_{1}$, energy of plastic deformation $E_{2}$, and energy of elastic deformation $E_{3}$, energy of compression $E_{\text {com }}$, which is the sum of $E_{2}$ and $E_{3}$, total energy $E_{\max }$ which is the sum of all energies, and plasticity $(11,12)$. 


\section{Measurement of tablet tensile strength}

Tensile strength of tablets was measured in 10 tablets no sooner than 24 hours after compression (tablets were stored in plastic tubes). Measurements were performed using a Schleuniger apparatus (Dr. Schleuniger Pharmatron AG, Switzerland), which measures the diameter and height of tablets with a precision of $0.01 \mathrm{~mm}$ and destruction force in N. Tensile strength of tablets was subsequently calculated according to the equation (Eq. 1) (13):

$$
P=2 F /(\pi d h)
$$

where $P$ is tensile strength of tablets in $\mathrm{MPa}, F$ is destruction force in $\mathrm{N}, d$ is tablet diameter in $\mathrm{mm}, h$ is tablet height in $\mathrm{mm}$.

\section{Mathematical and statistical processing of results}

The results of tensile strengths of tablets were statistically processed by means of the computer programs Excel and Qcexpert (TriloByte Statistical Software, s.r.o., Czech Republic). The energy and plasticity values were statistically processed by the computer program testXpert V 9.01 (Zwick GmbH\&Co, Germany) directly during compaction. In the case of similar significance of values, an ANOVA test at a level of significance of 0.05 was employed.

\section{RESULTS AND DISCUSSION}

The aim of the paper is to evaluate the compressibility and compactibility of directly compressible tableting materials containing the active ingredient tramadol hydrochloride, two types of coprocessed dry binders and two types of hypromellose differing in the viscosity degree. The coprocessed dry binders employed were Prosolv ${ }^{\circledR}$ SMCC 90, which is silicified microcrystalline cellulose with $2 \%$ of colloidal magnesium dioxide, and Disintequik ${ }^{\mathrm{TM}}$ MCC 25, which contains $75 \%$ of $\alpha$-lactose monohydrate and $25 \%$ of microcrystalline cellulose. In the function of hypromellose, i.e., the substance decelerating the release of the active ingredient, Methocel ${ }^{\mathrm{TM}}$ Premium K4M and Methocel $^{\mathrm{TM}}$ Premium K100M in concentrations of 30 and $50 \%$ were used. The lubricant employed in all tableting materials was magnesium stearate in a concentration of $1 \%$. Compressibility was evaluated by means of the energy profile of the compression process and compactibility by tensile strength of tablets. The compression force of $5 \mathrm{kN}$ was selected in such a way as to maintain the strength of the compacts from the tableting materials with both coprocessed dry binders higher than the lower limit of the optimal tensile strength of tablets, which is $0.56 \mathrm{MPa}$ (14).

\section{Energy profile of compression process}

Values of the energy profile of the compression process at the compression force of 5 $\mathrm{kN}$ are shown in Tables II and III. Values of the tableting materials with Prosolv SMCC 90 are presented in Table II and the values for the tableting materials with Disintequik MCC 25 are given in Table III. In the tableting materials without the active ingredient, the values of the total energy $E_{\max }$ are higher for the tableting materials with Prosolv SMCC 90, where 
there is no statistically significant difference between them $(p=0.094)$. In the case of Disintequik MCC 25, there is no clear difference between the values within the same concentration of different types of Methocel (30\% of Methocel $p=0.054 ; 50 \%$ of Methocel $p=$ 0.143). A more marked difference in values is observed in the use of the same type of Methocel in different concentrations, when $50 \%$ concentration of Methocel in the tableting material produces higher values of $E_{\max }$ than the $30 \%$ concentration. Tramadol in the tableting materials with Proslov SMCC 90 decreases the $E_{\max }$ values, but there is no marked difference in the values within the type and concentration of hypromellose employed $(p=$ 0.099). In the case of tableting materials with Disintequik MCC 25, the $E_{\max }$ values are less decreased by the action of tramadol and the influence of different concentrations of hypromellose is visible identically as in the tableting materials without the active ingredient, which means that values are always higher in the concentration of $50 \%$ of hypromellose; no difference is again observed within the type of hypromellose (30\% of Methocel $p=$ $0.373 ; 50 \%$ of Methocel $p=0.191$ ).

Values of the energy of precompression $E_{1}$ in the tableting materials without the active ingredient are higher and more equalized in the case of Prosolv SMCC 90. In the case of Disintequik MCC 25, the same influence is observed as in the $E_{\max }$ values, which means higher values for a $50 \%$ concentration of both types of Methocel and an insignificant difference within the type of Methocel used (30\% of Methocel $p=0.074 ; 50 \%$ of Methocel $p=$ 0.089). As far as the tableting materials with tramadol are concerned, the $E_{1}$ values in the tableting materials with Prosolv SMCC 90 are higher and more equalized than in the tableting materials with Disintequik MCC 25; tramadol decreases them slightly. In the case of tableting materials with Disintequik MCC 25, higher values are seen again in the case of a $50 \%$ concentration of both Methocel types and the addition of tramadol slightly decreases them. Another parameter under evaluation was the energy of compression $E_{\text {com }}$ which includes the energy accumulated in the tablet after compression, or the energy of plasticity $\left(E_{2}\right)$ and also the energy released during the decompression, or the energy of

Table II. Tableting materials with Prosolv SMCC 90: values of energy profiles of compression and plasticity

$$
(\mathrm{n}=10)
$$

\begin{tabular}{ccccccc}
\hline $\begin{array}{c}\text { Tableting } \\
\text { material }\end{array}$ & $E_{\max } \pm \mathrm{SD}(\mathrm{J})$ & $E_{1} \pm \mathrm{SD}(\mathrm{J})$ & $E_{2} \pm \mathrm{SD}(\mathrm{J})$ & $E_{3} \pm \mathrm{SD}(\mathrm{J})$ & $E_{\text {com }} \pm \mathrm{SD}(\mathrm{J})$ & $\mathrm{PI} \pm \mathrm{SD}(\%)$ \\
\hline 1 & $11.24 \pm 0.34$ & $6.09 \pm 0.33$ & $4.51 \pm 0.05$ & $0.65 \pm 0.01$ & $5.16 \pm 0.05$ & $87.40 \pm 0.12$ \\
2 & $10.81 \pm 0.14$ & $6.06 \pm 0.12$ & $4.09 \pm 0.04$ & $0.67 \pm 0.01$ & $4.75 \pm 0.04$ & $85.99 \pm 0.06$ \\
3 & $10.88 \pm 0.44$ & $5.69 \pm 0.40$ & $4.54 \pm 0.04$ & $0.65 \pm 0.01$ & $5.19 \pm 0.04$ & $87.50 \pm 0.11$ \\
4 & $10.69 \pm 0.20$ & $5.90 \pm 0.21$ & $4.12 \pm 0.04$ & $0.67 \pm 0.01$ & $4.79 \pm 0.04$ & $86.00 \pm 0.11$ \\
5 & $12.81 \pm 0.30$ & $6.68 \pm 0.29$ & $5.47 \pm 0.02$ & $0.66 \pm 0.01$ & $6.13 \pm 0.02$ & $89.26 \pm 0.06$ \\
6 & $12.68 \pm 0.18$ & $6.97 \pm 0.17$ & $5.02 \pm 0.04$ & $0.69 \pm 0.01$ & $5.71 \pm 0.04$ & $88.00 \pm 0.19$ \\
7 & $13.04 \pm 0.08$ & $6.95 \pm 0.10$ & $5.42 \pm 0.05$ & $0.66 \pm 0.01$ & $6.08 \pm 0.06$ & $89.16 \pm 0.05$ \\
8 & $12.79 \pm 0.16$ & $7.12 \pm 0.16$ & $4.99 \pm 0.03$ & $0.68 \pm 0.01$ & $5.67 \pm 0.03$ & $88.01 \pm 0.09$ \\
\hline
\end{tabular}

$E_{\max }$ - total energy; $E_{1}$ - energy of precompression; $E_{2}$ - energy of plastic deformation; $E_{3}$ - energy of elastic deformation; $E_{\text {com }}$ - energy of compression; Pl - plasticity; SD - standard deviation 
Table III. Tableting materials with Disintequik MCC 25: values of energy profiles of compression and plasticity $(\mathrm{n}=10)$

\begin{tabular}{ccccccc}
\hline $\begin{array}{c}\text { Tableting } \\
\text { material }\end{array}$ & $E_{\max } \pm \mathrm{SD}(\mathrm{J})$ & $E_{1} \pm \mathrm{SD}(\mathrm{J})$ & $E_{2} \pm \mathrm{SD}(\mathrm{J})$ & $E_{3} \pm \mathrm{SD}(\mathrm{J})$ & $E_{\text {com }} \pm \mathrm{SD}(\mathrm{J})$ & $\mathrm{PI} \pm \mathrm{SD}(\%)$ \\
\hline 9 & $7.24 \pm 0.14$ & $3.50 \pm 0.13$ & $3.14 \pm 0.04$ & $0.60 \pm 0.01$ & $3.74 \pm 0.04$ & $83.93 \pm 0.16$ \\
10 & $8.57 \pm 0.21$ & $4.68 \pm 0.19$ & $3.26 \pm 0.04$ & $0.64 \pm 0.01$ & $3.90 \pm 0.04$ & $83.59 \pm 0.13$ \\
11 & $7.29 \pm 0.12$ & $3.58 \pm 0.10$ & $3.11 \pm 0.03$ & $0.60 \pm 0.01$ & $3.71 \pm 0.03$ & $83.83 \pm 0.17$ \\
12 & $8.79 \pm 0.15$ & $4.85 \pm 0.13$ & $3.30 \pm 0.03$ & $0.64 \pm 0.01$ & $3.94 \pm 0.03$ & $83.81 \pm 0.16$ \\
13 & $7.90 \pm 0.22$ & $3.56 \pm 0.18$ & $3.75 \pm 0.04$ & $0.59 \pm 0.01$ & $4.34 \pm 0.04$ & $86.46 \pm 0.10$ \\
14 & $9.25 \pm 0.13$ & $4.84 \pm 0.13$ & $3.79 \pm 0.02$ & $0.63 \pm 0.01$ & $4.41 \pm 0.02$ & $85.73 \pm 0.05$ \\
15 & $8.26 \pm 0.11$ & $3.87 \pm 0.10$ & $3.80 \pm 0.01$ & $0.59 \pm 0.01$ & $4.38 \pm 0.01$ & $86.60 \pm 0.06$ \\
16 & $9.45 \pm 0.14$ & $5.08 \pm 0.15$ & $3.74 \pm 0.02$ & $0.63 \pm 0.01$ & $4.37 \pm 0.02$ & $85.57 \pm 0.13$ \\
\hline
\end{tabular}

$E_{\max -}$ total energy; $E_{1}$ - energy of precompression; $E_{2}$ - energy of plastic deformation; $E_{3}$ - energy of elastic deformation;

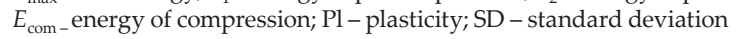

elasticity $\left(E_{3}\right)$. In the tableting materials without active ingredients, higher values are observed in the tableting materials with Prosolv SMCC 90 and this time higher values are observed in these tableting materials for $30 \%$ concentration of both types of Methocel, the same concentration of both types of Methocel showing practically the same values (30\% of Methocel $p=0.231 ; 50 \%$ of Methocel $p=0.266$ ). In the tableting materials with Disintequik MCC 25, no statistically significant difference was recorded between the $E_{\text {com }}$ values in the case of Methocel K100M ( $p=0.293)$. The $E_{\text {com }}$ values in the tableting materials with Prosolv SMCC 90 are decreased by tramadol, but in both cases they are higher for the 30 $\%$ concentration of both types of Methocel. The $E_{\text {com }}$ values for the tableting materials with Disintequik MCC 25 are again lower for the tableting materials with tramadol. Higher values are observed in the mixtures containing Methocel in the $50 \%$ concentration, this influence not being observed in the tableting materials without the active ingredient. Plasticity values in the tableting materials with Disintequik MCC 25 are slightly lower as well as the values with $50 \%$ of both types of Methocel in the tableting materials with both types of dry binders. Plasticity in the tableting materials with Prosolv SMCC 90 and tramadol shows slightly lower values than in the tableting materials without the active ingredient; the values are also slightly higher in the presence of $30 \%$ of both types of Methocel. Tableting materials with Disintequik MCC 25 and tramadol also possess slightly lower values of plasticity than the tableting materials without the active ingredient, but different effects of Methocel K100M concentrations are not observed in them $(p=0.393)$.

\section{Tensile strength of tablets}

The tensile strength values of tablets are shown in Figs. 1-3. Fig. 1 compares these values for all tableting materials without the active ingredient. The Figure shows lower 


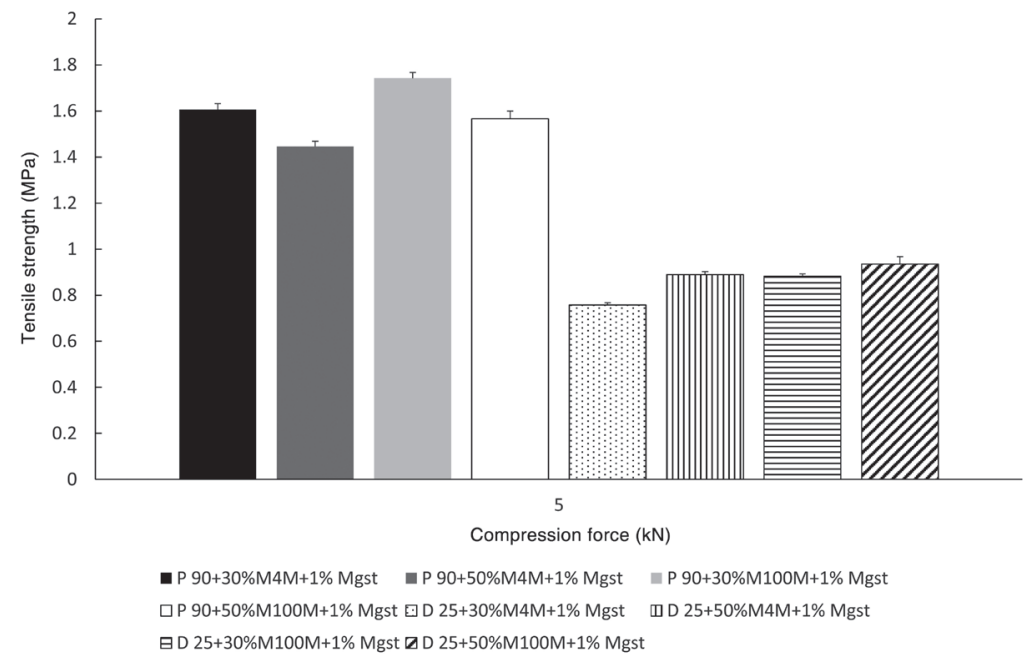

Fig. 1. Tensile strength of tablets at the compression force of $5 \mathrm{kN}(n=10)$ : Tableting materials without active ingredient. P 90 - Prosolv SMCC 90; D 25 - Disintequik MCC 25; M4M - Methocel K4M Premium CR; M100M - Methocel K100M Premium CR; Mgst - magnesium stearate.

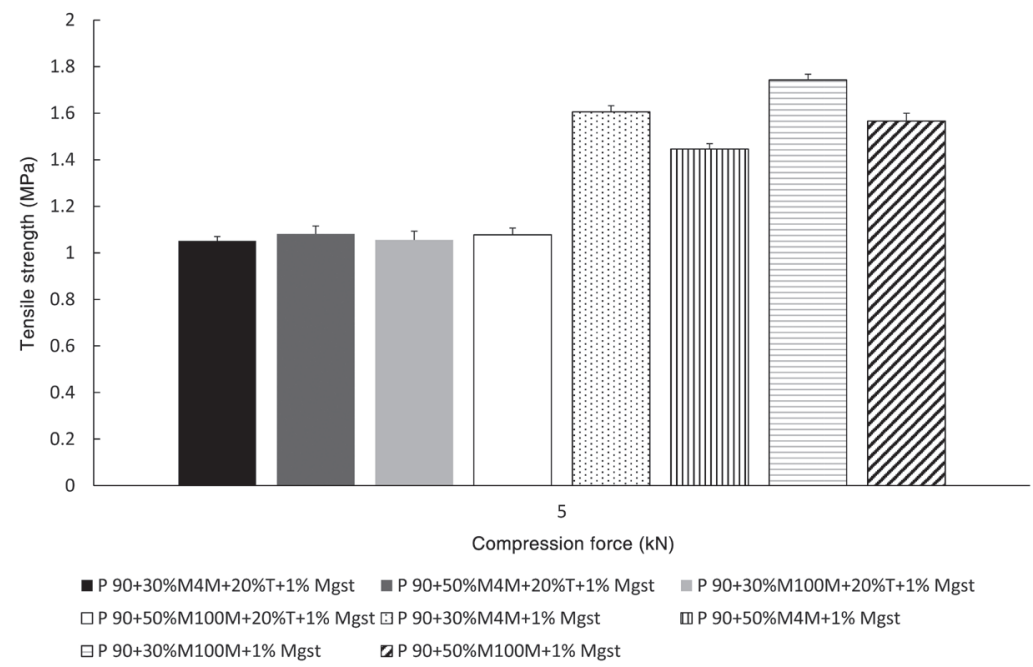

Fig. 2. Tensile strength of tablets at the compression force of $5 \mathrm{kN}(n=10)$ : Tableting materials with Prosolv SMCC 90. P 90 - Prosolv SMCC 90; M4M - Methocel K4M Premium CR; M100M - Methocel K100M Premium CR; Mgst - magnesium stearate; T - tramadol.

strength of tablets with Disintequik MCC 25. The tablets are stronger with a higher viscosity degree of hypromellose, i.e., Methocel Premium K100M, in the case of both coprocessed dry binders. The opposite effect of concentrations of both types of Methocel in combina- 


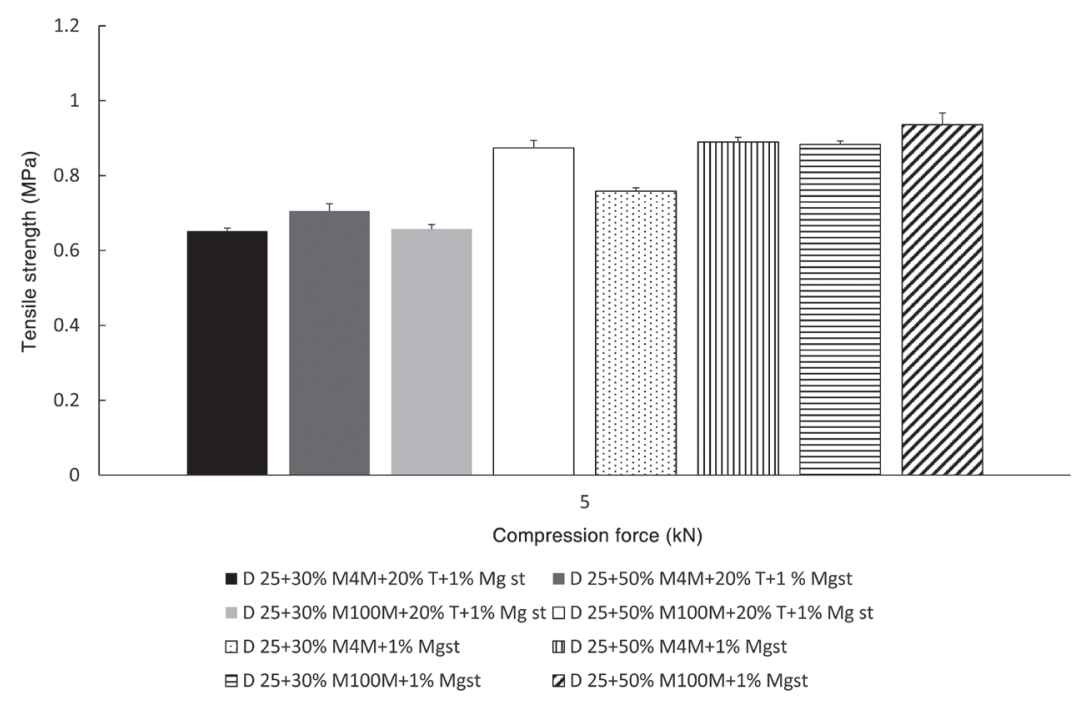

Fig. 3. Tensile strength of tablets at the compression force of $5 \mathrm{kN}(n=10)$ : Tableting materials with Disintequik MCC 25. D 25 - Disintequik MCC 25; M4M - Methocel K4M Premium CR; M100M Methocel K100M Premium CR; Mgst - magnesium stearate; T - tramadol.

tion with the employed coprocessed dry binders is interesting. Thus, in the case of Prosolv SMCC 90, the tablets with a $30 \%$ concentration of both types of Methocel are stronger, whereas in the case of Disintequik MCC 25, tablets with a $50 \%$ concentration of both types of Methocel are stronger. The strength of tablets of all tableting materials with Prosolv SMCC 90 is represented in Fig. 2. This figure shows a decrease in the strength of tablets due to the addition of tramadol, but also equalization of the strength of tablets for all compositions used $(p=0.252)$. In the case of the tableting materials with Disintequik MCC 25, there is also a decrease in the strength of tablets by the action of tramadol, tablets with 50 $\%$ of both types of Methocel remaining stronger (Fig. 3).

\section{CONCLUSIONS}

Tableting materials with Proslov SMCC 90 were better compressible and showed better compaction and consolidation properties of particles than tableting materials with Disintequik MCC 25. The values of total energy of compression, plasticity and tensile strength of tablets were higher in the tableting materials with Prosolv SMCC 90. Tramadol decreased these values and in the tableting materials with Prosolv SMCC 90 it equalized the values of tablet strength for all compositions tested. Tablets were stronger with a higher viscosity degree of hypromellose, i.e., Methocel Premium K100M, in the case of both dry binders.

Acknowledgments. - The study was supported by the firms JRS Pharma, Kerry and Colorcon $\mathrm{GmbH}$, which supplied the samples of the excipients tested. 


\section{REFERENCES}

1. P. Colombo, P. Santi et al., Swellable and Rigid Matrices: Controlled Release Matrices with Cellulose Ethers, in Pharmaceutical Dosage Forms: Tablets (Eds. L. L. Augsburger, S. W. Hoag), Informa Healthcare USA, Inc., New York 2008, pp. 433-468.

2. B. A. C. Carlin, Direct Compression and the Role of Filler Binders, in Pharmaceutical Dosage Forms: Tablets (Eds. L. L. Augsburger and S. W. Hoag), $3^{\text {th }}$ ed., Vol. 2, Informa Healthcare USA, Inc., New York 2008, pp. 173-216.

3. P. Gupta, S. T. Nachaegari and A. K. Bansal, Improved Excipients Functionality by Coprocessing, in Excipient Development for Pharmaceutical, Biotechnology and Drug Delivery Systems (Eds. A. Katdare, M. V. Chaubal), Informa Healthcare USA, Inc., New York 2006, pp. 109-126.

4. S. Kamel, N. Ali, K. Jahangir, S. M. Shah and A. A. El-Gendy, Pharmaceutical significance of cellulose: A Review, xXPRESS Polymer Letters 2 (2008) 758-778; DOI: 10.3144/expresspolymlett.2008.90.

5. K. Kachrimanis, I. Nikolakakis and S. Malamataris, Tensile strength and disintegration of tableted silicified microcrystalline cellulose: Influences of interparticle bonding, J. Pharm. Sci. 92 (2003) 1489-1501; DOI: 10.1002/jps.10403.

6. J. A. Zeleznik and J. Renak, High Functionality Excipients (HFE) - PROSOLV ${ }^{\circledR}$ SMCC as an effective strategy for generic drug formulation, Business Briefing, Pharmagenerics, (2004) 1-4.

7. B. E. Sherwood and J. W. Becker, A new class of high-functionality excipients: Silicified microcrystalline cellulose, Pharm. Tech. 22 (1998) 77-88.

8. KERRY, Disintequik ${ }^{\mathrm{TM}}$ MCC 25, Product document; http://www.sheffieldbioscience.com/disintequik_MCC25/; access date March 28, 2015.

9. J. S. M. Garr and M. H. Rubinstein, Compaction properties of cellulose-lactose direct-compression excipient, Pharm. Technol. Int. 3 (1991) 24-27.

10. R. J. Hardwood, Hydroxypropyl Methylcellulose, in Handbook of Pharmaceutical Excipients (Eds. A. H. Kibe, A. Wade et al.), American Pharmaceutical Association and Pharmaceutical Press, Washington 2000, pp. 252-255.

11. G. Ragnarsson, Force-displacement and Network Measurements, in Pharmaceutical Powder Compaction Technology (Eds. G. Alderborn and Ch. Nyström), Marcel Dekker Inc., New York 1996, pp. 77-96.

12. A. Stamm and C. Mathis, Verpressbarkeit von festen Hilfsstoffen für Direkttablettierung, Acta Pharm. Technol. 22 (1976) 7-16.

13. J. T. Fell and J. M. Newton, Determination of tablet strength by diametral compression test, J. Pharm. Sci. 59 (1970) 688-691; DOI: 10.1002/jps.2600590523.

14. V. A. Belousov, Choice of optimal pressure values in tableting medicinal powders, Khim. Farm. Zh. 10 (1976) 105-111. 